\title{
Modelling dust production in AGB stars
}

\author{
Flavia Dell'Agli ${ }^{1,2,3}$ \\ ${ }^{1}$ Instituto de Astrofísica de Canarias, Vía Láctea s/n, E-38200 La Laguna, Tenerife, Spain \\ ${ }^{2}$ Departamento de Astrofísica, Universidad de La Laguna (ULL), E-38206 La Laguna, Spain \\ ${ }^{3}$ Dipartimento di Fisica, Università di Roma "La Sapienza", P.le Aldo Moro 5, 00143, Roma, \\ Italy
}

\begin{abstract}
Asymptotic giant branch (AGB) stars are among the most important gas and dust polluters of the Universe. The latest AGB evolutionary models take into account dust production in the circumstellar envelope of these stars, starting from a detailed computation of the main physical processes and chemical surface variations occurring in this evolutionary phase. Following the formation and growth of dust particles, they provide the unique possibility of interpreting the AGB population observed in resolved galaxies. The first application was for the Spitzer observations of dusty AGBs in the Magellanic Clouds, characterising carbon-rich and oxygenrich stars in terms of initial mass, epoch of star formation, evolutionary time on the AGB and dust contribution. The same set of models are able to interpret the CNO surface abundances observed for the PNe of the same galaxies.
\end{abstract}

Keywords. Stars: abundances, AGB and post-AGB. ISM: dust, planetary nebulae: individual

\section{Dust production during the AGB evolution}

In this contribuiton we present the dust yields from low and intermediate mass stars, obtained considering the dust formation in AGB winds, starting from a self-consistent description of AGB evolution (see e.g. Ventura et al. 2014). This approach allows us to follow the evolution of the dust grain size distribution, optical properties of the wind and the rate of dust formation and to discuss the effects of the main physical processes involved in the AGB evolution: third drudge up (TDU) and hot bottom burning (HBB). The present analysis is used to interpret the infrared excess observed in AGB stars, and to infer their role in the dust pollution of the host environment; on the other hand, the comparison with the observations is important to draw information on the still poorly known physical mechanisms affecting the description of the AGB phase.

The evolutionary models are calculated with the ATON code (Canuto \& Mazzitelli 1999) from the pre-main sequence up to the end of the AGB phase; the range of masses explored is $1 M_{\odot} \leqslant M \leqslant 8 M_{\odot}$ with metallicities $3 \times 10^{-4} \leqslant Z \leqslant 18 \times 10^{-3}$. The main physical (luminosity, effective temperature, mass-loss rate) and chemical (surface abundances) output of the evolutionary computations are used as input to calculate dust formation and wind dynamics, following the model proposed by Ferrarotti \& Gail (2006).

In Fig. 1 we show the total amount of dust produced by AGB stars of different metallicities. Stars with masses below $3-4 M_{\odot}$, after several TDU episodes, increase their carbon surface abundance and eventually become carbon stars $(\mathrm{C} / \mathrm{O}>1)$. In a carbon-rich environment the main dust species that can be formed are silicon carbide ( $\mathrm{SiC}$ ), closer to the surface of the star, and solid carbon, in a more external region. Carbon grain sizes span the range $0.1 \mu m \leqslant a_{C} \leqslant 0.25 \mu m$, while $\mathrm{SiC}$ particles are smaller $(\sim 0.07 \mu m)$. Dust production depends on the availability of carbon molecules, therefore on the efficiency of TDU. More massive stars reach large temperatures $(>30$ million $\mathrm{K}(\mathrm{MK}))$ at the base of the convective envelope, thus being exposed to $\mathrm{HBB}$, with the consequent depletion 


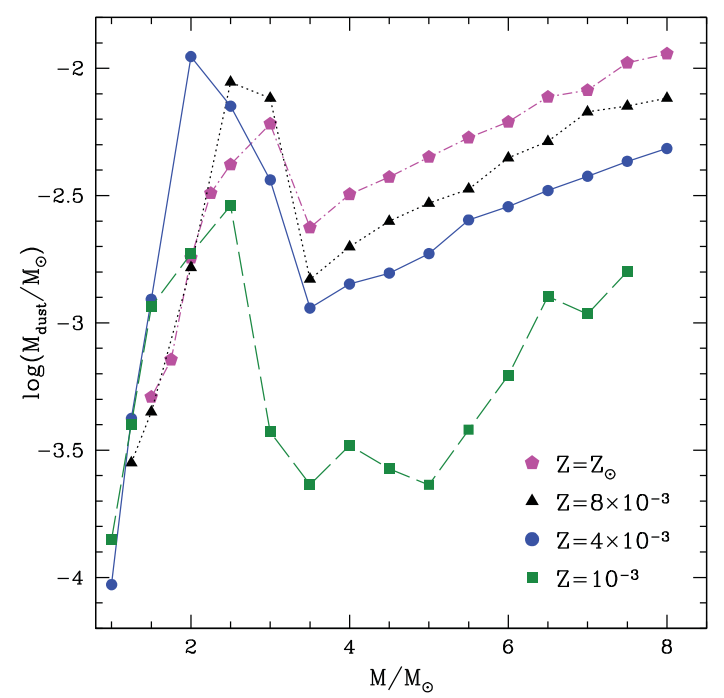

Figure 1. Total dust mass produced as a function of the initial mass and metallicity models.

of the surface carbon $(\mathrm{C} / \mathrm{O}<1)$ and increase in the nitrogen abundances. In an oxygenrich environment, the main dust compounds formed are silicates (olivine, pyroxene and quartz) and alumina dust $\left(\mathrm{Al}_{2} \mathrm{O}_{3}\right)$. Alumina dust grains form in an internal layer of the circumstellar envelope with respect to silicates; however, silicates reach larger dimensions (0.07-0.13 $\mu \mathrm{m})$ compared to alumina dust $(0.05-0.07 \mu \mathrm{m})$. Higher metallicity stars produce more dust, owing to the larger amount of silicon available for condensation. More massive stars undergo a more efficient HBB: this favours higher mass-loss rates, which increase the density of the wind and the dust formation rate.

\section{Modelling vs. observations}

We use the wide Spitzer-SAGE surveys of AGB stars in the Magellanic Clouds (Riebel et al. 2012, Boyer et al. 2011) to test the theoretical predictions and to assess the role played by AGB stars in polluting the host environment where they form. By using the DUSTY code (Nenkova et al. 1999), we follow the variation of the synthetic emerging spectra during the AGB phase for various masses and metallicities, starting from the outputs of the AGB+dust models. It is possible to construct evolutionary tracks, to reproduce the evolution of these objects in the colour-colour and colour-magnitude diagrams (CCD and CMD, respectively). In order to obtain a quantitive description of the AGB contribution to the total dust budget, we based the analysis on a population synthesis approach, using the star formation history $(\mathrm{SFH})$ and age-metallicity relations by Harris \& Zaritsky $(2004,2009)$. The comparison with the observations allows a full characterisation of the AGB stars in these galaxies, in terms of mass, formation epoch and chemical composition of the progenitors, and of the dust nowadays present in the circumstellar envelope (Dell'Agli et al. 2015a,b).

\subsection{AGB stars in the $M C s$}

The two panels of Fig. 2 show the CCDs of the AGB population of the LMC and SMC, in the Spitzer bands (Riebel et al. 2012, Boyer et al. 2011). We present some of the evolutionary tracks, which better represent the main protagonists of the SFH and dust pollution in each galaxy. 
In the LMC we can identify three main groups of stars, which occupy well defined regions of the CCD. The obscured carbon stars (OCS) and the hot bottom burning stars (HBBS), which constitute only the $\sim 20 \%$ and $\sim 1 \%$ of the total sample, respectively, are responsible for almost the totality of the dust pollution. The third group is a miscellaneous group of carbon and oxygen-rich stars (CMS) and represents the majority of the AGB population of the LMC; it is mainly composed of low-mass stars $\left(<3 M_{\odot}\right)$, with a low metallicity component $\left(Z<4 \times 10^{3}\right)$ and it is characterised by a low infrared excess.

The OCS group is entirely composed of carbon stars, populating a diagonal band in the CCD, with $0.2<[3.6]-[4.5]$ and $0.2<[5.6]-[8.0]<1.6$. They are the progeny of stars with mass $1 M_{\odot} \leqslant M \leqslant 3 M_{\odot}$, and formed $3 \times 10^{8}-3 \times 10^{9}$ years ago. As more carbon is accumulated on the surface, the stars produce more dust, moving towards redder colours, with a corresponding increase in the optical depth $\left(0.01<\tau_{10}<3.5\right)$. The stars formed during the peak in the SFH which occurred $\sim 5 \times 10^{8}$ year ago, and descend from stars with initial mass in the range $2 M_{\odot} \leqslant M \leqslant 3 M_{\odot}$ of higher metallicity $\left(Z \geqslant 4 \times 10^{3}\right)$ and are the only ones which evolve redder than $[3.6]-[4.5]>1$.

Stars clustering around $[3.6]-[4.5] \sim 0.2,[5.8]-[8.0] \sim 0.8$ belong to the HBBS group. These stars have initial masses in the range $3.5 M_{\odot} \leqslant M \leqslant 7.5 M_{\odot}$ and are experiencing $\mathrm{HBB}$, forming mainly silicates and alumina dust. The lower extinction coefficients of silicates compared to carbon dust determine a smaller optical depth of the circumstellar envelope in these stars ( $\tau_{10}$ is below 2 in all cases) compared to their C-rich counterparts. They belong to the more metal-rich population $\left(Z \geqslant 4 \times 10^{3}\right)$ and formed during the burst in the SFH which occurred $\sim 10^{8}$ years ago (Harris \& Zaritsky 2009).

In the SMC we distinguish a population of stars with negligible infrared emission, similar to the CMS group in the LMC. It is composed mainly of oxygen-rich, low mass stars of various metallicities, in the AGB phases previous to or at the beginning of the C-star phase. In analogy to the LMC case, in the SMC we identify a sequence of dusty AGB stars with infrared emission that extends diagonally towards the reddest region of the CCD. We interpret this group of stars, with [3.6] $-[4.5]>0.2$, as C-rich objects producing mainly carbon dust. The majority of the stars in this group have initial mass $1.5-1.75 M_{\odot}$ and metallicity $Z=4 \times 10^{-3}$, formed between $1-1.5 \mathrm{Gyr}$ ago. They correspond to the objects with the largest optical depth, formed during the secondary peak in the SFH of the SMC. The comparison between the evolutionary tracks of the $1.5 M_{\odot}$ model of $Z=4 \times 10^{3}$ and the $3 M_{\odot}$ model of $Z=8 \times 10^{3}$ shows that the latter evolves to much redder colours, which is the reason for the difference in the extension of the C-star sequences observed in the LMC and SMC. Turning to more massive stars, unlike the LMC, the SFR of the SMC presents a minimum at $\sim 100 \mathrm{Myr}$, when stars with initial mass $3.5 M_{\odot} \leqslant M \leqslant 7.5 M_{\odot}$ formed. This explain the paucity of AGB stars in the region around $[3.6]-[4.5] \sim 0.2,[5.8]-[8.0] \sim 0.8$ in the SMC CCD diagram.

\subsection{Interpreting $C N O$ abundances in $M C s P N e$}

The final surface composition of the AGB models previously described have been recently used to characterize the MCs planetary nebulare (PNe) in terms of age, chemical composition and mass of the progenitors, on the base of the CNO abundances observed (Ventura et al. 2015, 2016). A dichotomy in the distribution of the nitrogen abundances is observed in both galaxies, a clear signature of the HBB activation in stars of initial mass above $\sim 3 M_{\odot}$. The objects enriched in nitrogen, with $\log (\mathrm{N} / \mathrm{H})+12 \geqslant 8$, are the progeny of stars with mass higher than the aforementioned threshold, and formed 50-200 Myr ago. PNe with $\log (N / H)+12<7.5$ are interpreted as the final stages of stars that did not experience any HBB. These objects, with initial mass below $\sim 3 M_{\odot}$, formed between 500 Myr and 10 Gyr ago, with a different spread in carbon determined by the 

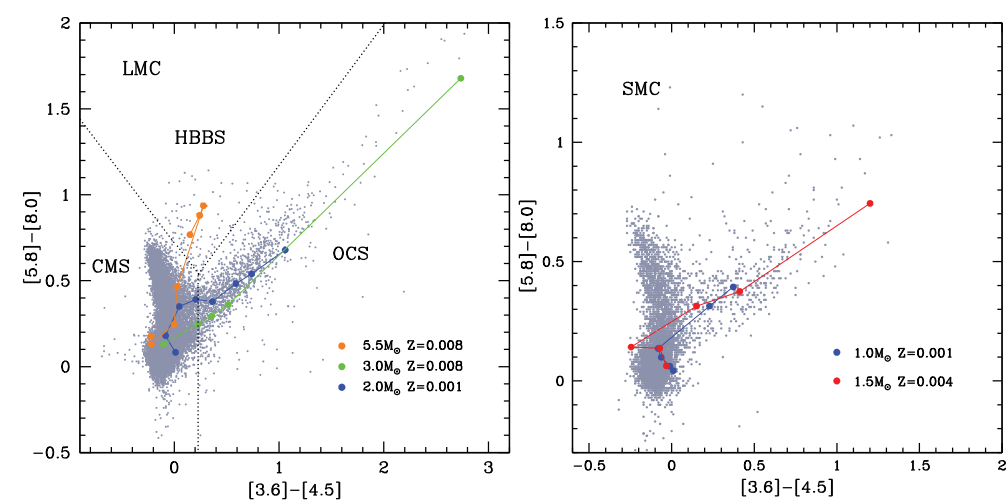

Figure 2. Evolutionary tracks of AGB stars of different initial mass and metallicity and the AGB stars observed in the LMC (left panel) from Riebel et al. (2012) and in the SMC (right panel) from Boyer et al. (2011).

different number of TDU episodes experienced. The highest carbon abundances found in both the samples is $\log (\mathrm{C} / \mathrm{H})+12<9$. If confirmed by future observations, it indicates a limited number of TDU episodes was experienced, probably due to a rapid loss of the external envelope.

\section{Conclusions}

We present models of AGB stars which consider the formation of dust in the circumstellar envelope and discuss the trend of the amount of dust produced with mass and metallicity. The results are compared with the infrared observations of the AGB stars in the MCs and with the chemical composition of PNe in the MCs. In both cases the agreement is satisfactory, adding more robustness to the present findings.

\section{Acknowledgments}

FD acknowledges support from LOC/SOC of the IAU323 and from "La Sapienza", University of Rome under the grant "Avvio alla ricerca 2015".

\section{References}

Boyer M. L., et al. 2011, AJ, 142, 103 et al. 2014a, MNRAS, 442, L38

Dell'Agli F., Ventura P., Schneider R., Di Criscienzo M., et al. 2015a, MNRAS, 447, 2992

Dell'Agli F., Ventura P., García-Hernández D. A., Schneider R. et al. 2015b, MNRAS, 454, 4235

Ferrarotti A. D. \& Gail H. P., 2006, A\&GA, 553, 576

Harris J., Zaritsky D. 2004, $A J, 127,1531$

Harris J., Zaritsky D. 2009, ApJ, 138, 1243

Mazzitelli I., D'Antona F., \& Ventura P., 1999, A\&A, 348, 846

Nenkova M., Ivezic Z., \& Elitzur M., 1999, in LPIContributions 969, 20

Riebel D., Srinivasan S., Sargent B., Meixner M. 2012, AJ, 753, 71

Ventura P., Dell'Agli F., Di Criscienzo M., Schneider R., et al. 2014a, MNRAS, 439, 977

Ventura P., Stanghellini L., Dell'Agli F., García-Hernández D. A., Di Criscienzo M. 2015, MNRAS, 452, 3679

Ventura P., Stanghellini L., Di Criscienzo M., García-Hernández D. A., Dell'Agli F. 2015, $M N R A S, 460,3940$

\section{Discussion}

Schoenberner: Can you say something about the mass-loss rate? 